\title{
MINIMIZING THE SUM OF PROJECTIONS OF A FINITE SET
}

\author{
VSEVOLOD F. LEV AND MISHA RUDNEV
}

\begin{abstract}
Consider the projections of a finite set $A \subset \mathbb{R}^{n}$ onto the coordinate hyperplanes. How small can the sum of the sizes of these projections be, given the size of $A$ ? In a different form, this problem has been studied earlier in the context of edgeisoperimetric inequalities on graphs, and it is can be derived from the known results that there is a linear order on the set of $n$-tuples with non-negative integer coordinates, such that the sum in question is minimised for the initial segments with respect to this order. We present a new, self-contained and constructive proof, enabling us to obtain a stability result and establish algebraic properties of the smallest possible projection sum. We also solve the problem of minimising the sum of the sizes of the one-dimensional projections.
\end{abstract}

\section{Preliminaries}

Given an integer $n \geq 1$, for each $i \in[1, n]$ denote by $\pi_{i}$ the orthogonal projection of the vector space $\mathbb{R}^{n}$ onto the coordinate hyperplane $\left\{\left(x_{1}, \ldots, x_{n}\right) \in \mathbb{R}^{n}: x_{i}=0\right\}$. For a finite set $A \subset \mathbb{R}^{n}$, as a simple consequence of the Loomis-Whitney inequality (see, for instance, [LW49, CGG16, GMR10]), we have

$$
\prod_{i=1}^{n}\left|\pi_{i}(A)\right| \geq|A|^{n-1} ;
$$

combining this estimate with the arithmetic mean-geometric mean inequality yields

$$
\sum_{i=1}^{n}\left|\pi_{i}(A)\right| \geq n|A|^{1-1 / n} .
$$

The Loomis-Whitney inequality is known to be sharp, turning into an exact equality when the set under consideration is an axes-aligned rectangular parallelepiped (and, in the convex set settings, only in this case, as it follows from the argument of [LW49]). In contrast, the estimate (1) is not sharp; say, it shows that for a three-dimensional, five-point set, the sum of the projection sizes is at least as large as $3 \cdot 5^{2 / 3} \approx 8.77$, while it is not difficult to see that, in fact, this sum cannot be smaller than 10. This leads naturally to the following question: exactly how small can the sum in the left-hand side

2010 Mathematics Subject Classification. Primary: 52A38; secondary: 52A40, 05A20.

Key words and phrases. Isoperimetric problem, Loomis-Whitney inequality, projections. 
of (1) be for a finite set $A \subset \mathbb{R}^{n}$ of given size? Loosely speaking, we want to know how much the points of an $n$-dimensional set of given size can hide behind each other.

The answer to a tightly related question is due to Bollobás and Leader [BL91, Theorem 15], where it was cast as the edge-isoperimetric problem for the $n$-dimensional grid graph; see also Harper [H04, Theorem 7.1] and historical comments [H04, page 142], as well as the references contained therein. Our goal here is to give a direct, independent, and self-contained solution of a discrete-geometric flavour, avoiding references to graph theory and making the underling rearrangement procedure maximally algorithmic. This enables us to prove a stability result showing that, in certain cases, the set with the smallest sum of the projection sizes is, essentially, unique. Furthermore, we establish some algebraic properties of the smallest possible value of this sum as a function of the size of the set being projected. Finally, in the Appendix we discuss and solve a similar problem for the one-dimensional projections.

\section{Summary OF RESUlts}

If $|A|=K^{n}$ with an integer $K \geq 1$, then (1) implies

$$
\sum_{i=1}^{n}\left|\pi_{i}(A)\right| \geq n K^{n-1},
$$

which is attained for the discrete $n$-dimensional cube $A=[0, K-1]^{n}$. The situation where $|A|$ is not a perfect $n$th power is much subtler and requires some preparation to discuss.

Denote by $\mathbb{N}_{0}$ the set of all non-negative integers. Following [BL91], we define the cube order on $\mathbb{N}_{0}^{n}$ by saying that $\left(x_{1}, \ldots, x_{n}\right)$ precedes $\left(y_{1}, \ldots, y_{n}\right)$ if there exists $l_{0} \in \mathbb{N}_{0}$ and $j \in[1, n]$ such that $\left\{i \in[1, n]: x_{i}=l\right\}=\left\{i \in[1, n]: y_{i}=l\right\}$ for each $l>l_{0}$, and also $\left\{i \in[j+1, n]: x_{i}=l_{0}\right\}=\left\{i \in[j+1, n]: y_{i}=l_{0}\right\}$, while $x_{j}<y_{j}=l_{0}$. For integer $m \geq 0$, by $\mathcal{I}_{n}(m)$ we denote the length- $m$ initial segment of $\mathbb{N}_{0}^{n}$ with respect to the cube order; thus, for instance, $\mathcal{I}_{n}(0)=\varnothing, \mathcal{I}_{1}(m)=[0, m-1]$,

$$
\mathcal{I}_{2}(10)=\{(0,0),(1,0),(0,1),(1,1),(2,0),(2,1),(0,2),(1,2),(2,2),(3,0)\}
$$

and

$$
\begin{aligned}
& \mathcal{I}_{3}(17)=\{(0,0,0),(1,0,0),(0,1,0),(1,1,0),(0,0,1),(1,0,1),(0,1,1),(1,1,1), \\
&(2,0,0),(2,1,0),(2,0,1),(2,1,1),(0,2,0),(1,2,0),(0,2,1),(1,2,1),(2,2,0)\} .
\end{aligned}
$$

Speaking about initial segments we will always mean finite initial segments of $\mathbb{N}_{0}^{n}$ with respect to the cube order, with the value of $n$ determined by the context.

We notice that the cube order is quite similar, but not identical to the order introduced in [H04, Section 7.1.1]; in fact, the latter order is defined on the whole grid $\mathbb{Z}^{n}$. 
We say that the initial segment $I_{1}$ is shorter than the initial segment $I_{2}$ if $\left|I_{1}\right|<\left|I_{2}\right|$; equivalently, if $I_{1} \subset I_{2}$.

Informally, the initial segments fill in $\mathbb{N}_{0}^{n}$ cube-wise: once the cube $[0, K-1]^{n}$ has been filled in, for some integer $K \geq 1$, the $n$ faces

$$
0 \leq x_{1}, \ldots, x_{i} \leq K, x_{i+1}=K, 0 \leq x_{i+2}, \ldots, x_{n} \leq K-1, \quad i \in[0, n-1]
$$

are completed one by one to get a covering of the next cube $[0, K]^{n}$, etc. If $m=(K+$ $1)^{i} K^{n-i}$ with some $i \in[0, n-1]$, then

$$
\mathcal{I}_{n}(m)=\left\{\left(x_{1}, \ldots, x_{n}\right) \in \mathbb{N}_{0}^{n}: 0 \leq x_{1}, \ldots, x_{i} \leq K, 0 \leq x_{i+1}, \ldots, x_{n} \leq K-1\right\}
$$

is an axes-aligned rectangular parallelepiped; in this case we say that the segment $\mathcal{I}_{n}(m)$ is closed (the intuition behind this term will be clear from the next section). The edges of a closed initial segment are its orthogonal projections onto the coordinate axes; thus, for instance, the two-element initial segment has one edge of size 2 , all other edges being of size 1 .

In general, for any integer $m \geq 1$ there are uniquely defined integers $K \geq 1$ and $i \in[0, n-1]$ such that

$$
K^{n} \leq m<(K+1)^{n}
$$

and, indeed,

$$
(K+1)^{i} K^{n-i} \leq m<(K+1)^{i+1} K^{n-i-1}
$$

writing then

$$
m=(K+1)^{i} K^{n-i}+R, \quad 0 \leq R<(K+1)^{i} K^{n-1-i},
$$

the initial segment $\mathcal{I}_{n}(m)$ is the disjoint union of the closed initial segment $\mathcal{I}_{n}((K+$ $\left.1)^{i} K^{n-i}\right)$, which is the parallelepiped in the right-hand side of (2), and a translate of the $(n-1)$-dimensional initial segment $\mathcal{I}_{n-1}(R)$, contained in the hyperplane $x_{i+1}=K$.

We can now state our first principal result.

Theorem 1. For every integer $n \geq 1$ and every finite set $A \subset \mathbb{R}^{n}$, letting $m:=|A|$, we have

$$
\sum_{i=1}^{n}\left|\pi_{i}(A)\right| \geq \sum_{i=1}^{n}\left|\pi_{i}\left(\mathcal{I}_{n}(m)\right)\right| .
$$

In Section 5 we prove Theorem 1 in the particular case where $A \subset \mathbb{N}_{0}^{n}$; the general case then follows readily by observing that if $A \subseteq S^{n}$ with a finite set $S \subseteq \mathbb{R}$, then for any injective mapping $\varphi: S \rightarrow \mathbb{N}_{0}$, writing $B:=\varphi^{\otimes n}(A) \subset \mathbb{N}_{0}^{n}$ we have $|B|=|A|$ and $\left|\pi_{i}(A)\right|=\left|\pi_{i}(B)\right|$ for each $i \in[1, n]$. Indeed, this observation shows that the estimate of Theorem 1 remains valid when $A \subseteq S^{n}$ with a set $S$ of any nature, not necessarily contained in the set of real numbers (although in this case the projections $\pi_{i}$ must be redefined appropriately). 
We denote the left-hand side of (6) by $\sigma_{n}(A)$ and, with some abuse of notation, its right-hand side by $\sigma_{n}(m)$; that is, $\sigma_{n}(A)$ is the sum of the sizes of the projections of the finite set $A \subset \mathbb{R}^{n}$ onto the coordinate hyperplanes, and $\sigma_{n}(m)$ is this sum in the special case where the set in question is the length- $m$ initial segment. Thus, for instance,

$$
\sigma_{n}(0)=0, \sigma_{1}(m)=1 \text { if } m>0,
$$

and, as one can easily verify,

$$
\sigma_{2}(m)= \begin{cases}2 K+1 & \text { if } K^{2}<m \leq K(K+1), \\ 2 K+2 & \text { if } K(K+1)<m \leq(K+1)^{2}\end{cases}
$$

for any integer $K \geq 0$. Also, it follows from the explanation above that for $K, i$, and $R$ defined by (3) $-(5)$, we have

$$
\begin{aligned}
\sigma_{n}(m) & =\sigma_{n}\left((K+1)^{i} K^{n-i}\right)+\sigma_{n-1}(R) \\
& =(n K+n-i)(K+1)^{i-1} K^{n-i-1}+\sigma_{n-1}(R) ;
\end{aligned}
$$

along with (7), this relation gives a recursive, completely algebraic definition of the quantities $\sigma_{n}(m)$.

We now address the corresponding stability problem.

We say that a finite set $A \subset \mathbb{R}^{n}$ is a minimiser if its projection sum $\sigma_{n}(A)$ is smallest possible among all sets in $\mathbb{R}^{n}$ of size $|A|$. Thus, Theorem 1 says that every initial segment of $\mathbb{N}_{0}^{n}$ is a minimiser, but it is not true in general that any minimiser is an initial segment, or even is "similar" to an initial segment in some reasonable sense (see Section 6 for a rigorous definition of similarity). Say, for integer $K \geq C \geq 1$, the set $A:=[0, K-$ $C] \times[0, K+C] \subset \mathbb{N}_{0}^{2}$ is not an initial segment, while $|A|=(K+1)^{2}-C^{2}$ and therefore $\sigma_{2}(A)=2 K+2=\sigma_{2}(|A|)$ (cf. (8) ), showing in view of Theorem 1 that $A$ is a minimiser. In Section 6 we prove, however, that every closed initial segment is (up to similarity) a unique minimiser.

Theorem 2. Suppose that $n \geq 1$ is an integer. If $m=(K+1)^{i} K^{n-i}$ with integers $K \geq 1$ and $i \in[0, n-1]$, then every minimiser in $\mathbb{R}^{n}$ of size $m$ is a Cartesian product of $i$ real sets of size $K+1$, and $n-i$ real sets of size $K$.

The following lemma, proved in Section 4, is an important ingredient of the proof of Theorem 1.

Lemma 1. Let $n \geq 1$ be an integer.

i) Suppose that $I_{1}, I_{2}, J_{1}, J_{2} \subset \mathbb{N}_{0}^{n}$ are initial segments such that $\left|I_{1}\right|+\left|I_{2}\right|=\left|J_{1}\right|+\left|J_{2}\right|$, $\left|J_{1}\right| \leq\left|I_{1}\right| \leq\left|I_{2}\right| \leq\left|J_{2}\right|$, and $J_{2}$ is closed. Then

$$
\sigma_{n}\left(J_{1}\right)+\sigma_{n}\left(J_{2}\right) \leq \sigma_{n}\left(I_{1}\right)+\sigma_{n}\left(I_{2}\right)
$$


ii) If $I, I_{1}, I_{2} \subset \mathbb{N}_{0}^{n}$ are non-empty initial segments such that $|I|=\left|I_{1}\right|+\left|I_{2}\right|$, then

$$
\sigma_{n}(I)<\sigma_{n}\left(I_{1}\right)+\sigma_{n}\left(I_{2}\right)
$$

iii) If $n \geq 2$ and $I_{n-1} \subset \mathbb{N}_{0}^{n-1}, I_{n} \subset \mathbb{N}_{0}^{n}$ are non-empty initial segments such that $\left|I_{n-1}\right|=\left|I_{n}\right|$, then

$$
\sigma_{n}\left(I_{n}\right)>\sigma_{n-1}\left(I_{n-1}\right) .
$$

An "algebraic restatement" of Lemma 1 may be of interest.

Lemma $\mathbf{1}^{\prime}$. Let $n \geq 1$ be an integer.

i) Suppose that $l_{1} \leq m_{1} \leq m_{2} \leq l_{2}$ are non-negative integers such that $m_{1}+m_{2}=$ $l_{1}+l_{2}$. If $\mathcal{I}_{n}\left(l_{2}\right)$ is closed, then

$$
\sigma_{n}\left(l_{1}\right)+\sigma_{n}\left(l_{2}\right) \leq \sigma_{n}\left(m_{1}\right)+\sigma_{n}\left(m_{2}\right) .
$$

ii) If $m_{1}, m_{2} \geq 1$ are integers, then

$$
\sigma_{n}\left(m_{1}+m_{2}\right)<\sigma_{n}\left(m_{1}\right)+\sigma_{n}\left(m_{2}\right) .
$$

iii) If $n \geq 2$ and $m \geq 1$ is an integer, then

$$
\sigma_{n}(m)>\sigma_{n-1}(m)
$$

We remark that subadditivity established by Lemma 1 ii) and Lemma 1' ii) can be viewed as a combinatorial analogue of the well-known physical fact that merging two spherical droplets into one reduces the total surface area.

Our last result establishes yet another interesting algebraic property of the functions $\sigma_{n}$.

Theorem 3. For any integers $n, s \geq 1$ and $m_{1}, \ldots, m_{s} \geq 0$, we have

$$
\sigma_{n}\left(m_{1}+\cdots+m_{s}\right) \leq \sigma_{n-1}\left(m_{1}\right)+\cdots+\sigma_{n-1}\left(m_{s}\right)+\max \left\{m_{1}, \ldots, m_{s}\right\} .
$$

In Section 7 we derive Theorem 3 from Theorem 1 and, indeed, show that, somewhat unexpectedly, the two theorems are equivalent in the sense that each of them follows easily from the other one.

In the next section we introduce important notation and terminology used throughout. Having finished with this, we prove Lemma 1 in Section 4, and Theorems 1 and 2 in Sections 5 and 6, respectively. The equivalence of the former theorem and Theorem 3 is established in Section 7. Finally, in the Appendix we develop a similar theory for the one-dimensional projections, and in particular show that their sum is also minimised when the set under consideration is an initial segment.

The proofs are purely combinatorial, based on point rearrangements. 


\section{Notation AND TERminology}

For integer $1 \leq i \leq n$, we denote the coordinate vectors in $\mathbb{R}^{n}$ by $e_{1}, \ldots, e_{n}$, and write

$$
\mathcal{X}_{i}:=\operatorname{Sp}\left\{e_{i}\right\}
$$

for the coordinate axes, and

$$
\mathcal{L}_{i}:=\operatorname{Sp}\left\{e_{1}, \ldots, e_{i-1}, e_{i+1}, \ldots, e_{n}\right\}
$$

for the coordinate hyperplanes. The axis $\mathcal{X}_{n}$ will be referred to as vertical, and the corresponding hyperplane $\mathcal{L}_{n}$ and its translates, as well as the projection $\pi_{n}$, as horizontal.

The reader may find helpful to think of points $x \in \mathbb{Z}^{n}$ as unit cubes, with the base vertex at $x$, and visualize sets $A \subseteq \mathbb{Z}^{n}$ as built of such cubes.

The intersections of a set $A \subseteq \mathbb{Z}^{n}$ with horizontal hyperplanes will be called the slabs of $A$. If $n=1$, then the slabs are zero-dimensional; hence, either empty, or singletons. Notice that closed initial segments in $\mathbb{N}_{0}^{n}$ are stable under permuting non-empty slabs.

Recall that an initial segment of $\mathbb{N}_{0}^{n}$ is closed if it is an axes-aligned rectangular parallelepiped whose edges differ by at most 1 , and for all $1 \leq i<j \leq n$, the edge along $\mathcal{X}_{j}$ is not longer than the edge along $\mathcal{X}_{i}(\mathrm{cf} .(2))$.

Given an initial segment $I \subset \mathbb{N}_{0}^{n}$, we define its (strict) interior to be the longest closed initial segment (strictly) contained in $I$, and we denote the interior and the strict interior of $I$ by $\operatorname{int}(I)$ and $\operatorname{int}^{*}(I)$, respectively. Similarly we define the (strict) closure of $I$ to be the shortest closed initial segment (strictly) containing $I$, and denote the closure and the strict closure of $I$ by $\mathrm{cl}(I)$ and $\mathrm{cl}^{*}(I)$, respectively. The boundary of $I$ is defined by $\partial I:=I \backslash \operatorname{int}(I)$, and its strict boundary by $\partial^{*} I:=I \backslash \operatorname{int}^{*}(I)$; thus, the boundary is empty if and only if $I$ is closed, while the strict boundary is always nonempty whenever $I \neq \varnothing$. Boundaries can be treated either as $(n-1)$-dimensional sets embedded in $\mathbb{N}_{0}^{n}$, or as initial segments in $\mathbb{N}_{0}^{n-1}$.

We remark that for an initial segment $I \neq \varnothing$, any of the three conditions $\mathrm{cl}^{*}(I)=\operatorname{cl}(I)$, $\operatorname{int}^{*}(I)=\operatorname{int}(I)$, and $\partial^{*} I=\partial I$ is equivalent to $I$ not being closed.

As a version of (9), for any initial segment $I \subset \mathbb{N}_{0}^{n}$ with $|I|>1$, we have

$$
\sigma_{n}(I)=\sigma_{n}\left(\operatorname{int}^{*}(I)\right)+\sigma_{n-1}\left(\partial^{*} I\right) .
$$

(If $|I|=1$, then $\operatorname{int}^{*}(I)=\varnothing$ and the left-hand side of (11) exceeds by 1 its right-hand side.) This basic, but important identity allows us to argue inductively in the forthcoming proofs. It becomes evident upon observing that the strict boundary $\partial^{*} I$ is an $(n-1)$ dimensional set, attached to and not larger than a face of $\operatorname{int}^{*}(I)$ which we visualize as a rectangular parallelepiped; hence $\partial^{*} I$ does not contribute to the projection along the axis, normal to the face under consideration.

Notation-wise, we will occasionally use (11) in the form

$$
\sigma_{n}(|I|)=\sigma_{n}\left(\left|\operatorname{int}^{*}(I)\right|\right)+\sigma_{n-1}\left(\left|\partial^{*} I\right|\right),
$$


the equivalence following from the fact that $\operatorname{int}^{*}(I)$ and $\partial^{*} I$ are initial segments (in $\mathbb{N}_{0}^{n}$ and $\mathbb{N}_{0}^{n-1}$, respectively).

\section{Proof of Lemma 1}

We use induction by $n$. The base case $n=1$ is easy to verify, and we proceed assuming that $n \geq 2$. We first prove iii), then i), and, finally, ii).

Addressing iii), we use (the inner) induction by the common size $m$ of the initial segments $I_{n-1}$ and $I_{n}$. For $m=1$ the estimate follows from

$$
\sigma_{n}\left(I_{n}\right)=n=\sigma_{n-1}\left(I_{n-1}\right)+1 .
$$

For $m \geq 2$, by (11) and the induction hypothesis, we have

$$
\sigma_{n}\left(I_{n}\right)=\sigma_{n}\left(\left|\operatorname{int}^{*}\left(I_{n}\right)\right|\right)+\sigma_{n-1}\left(\left|\partial^{*} I_{n}\right|\right)>\sigma_{n-1}\left(\left|\operatorname{int}^{*}\left(I_{n}\right)\right|\right)+\sigma_{n-1}\left(\left|\partial^{*} I_{n}\right|\right) .
$$

Applying ii) inductively, we see that the right-hand side is larger than

$$
\sigma_{n-1}\left(\mid \text { int }^{*}\left(I_{n}\right)|+| \partial^{*} I_{n} \mid\right)=\sigma_{n-1}\left(\left|I_{n}\right|\right)=\sigma_{n-1}\left(\left|I_{n-1}\right|\right)=\sigma_{n-1}\left(I_{n-1}\right) .
$$

This completes the proof of iii), and we now turn to i).

If $J_{1}=\varnothing$, then the assertion follows from ii), and we thus assume that $J_{1} \neq \varnothing$, implying $I_{1} \neq \varnothing$. Our plan is to shorten $I_{1}$ and lengthen $I_{2}$, while keeping the sum $\left|I_{1}\right|+\left|I_{2}\right|$ intact, to get, after a number of iterations, to the situation where $I_{2}=J_{2}$. Formally, we act as follows.

Let

$$
\delta:=\min \left\{\left|\partial^{*} I_{1}\right|,\left|\mathrm{cl}^{*}\left(I_{2}\right) \backslash I_{2}\right|\right\}
$$

(the number of elements to be transferred from $I_{1}$ to $I_{2}$ ), and define $I_{1}^{\prime}$ and $I_{2}^{\prime}$ to be the initial segments of $\mathbb{N}_{0}^{n}$ of sizes $\left|I_{1}^{\prime}\right|=\left|I_{1}\right|-\delta$ and $\left|I_{2}^{\prime}\right|=\left|I_{2}\right|+\delta$. Notice that $\delta>0$, and since $J_{2}$ is closed, if $I_{2} \neq J_{2}$, then we have $\mathrm{cl}^{*}\left(I_{2}\right) \subseteq J_{2}$, implying $\delta \leq\left|J_{2} \backslash I_{2}\right|$. Consequently, $\left|I_{2}^{\prime}\right|=\left|I_{2}\right|+\delta \leq\left|J_{2}\right|$, whence $\left|I_{1}^{\prime}\right|=\left|J_{1}\right|+\left|J_{2}\right|-\left|I_{2}^{\prime}\right| \geq\left|J_{1}\right|$.

We now prove that

$$
\sigma_{n}\left(I_{1}^{\prime}\right)+\sigma_{n}\left(I_{2}^{\prime}\right) \leq \sigma_{n}\left(I_{1}\right)+\sigma_{n}\left(I_{2}\right) .
$$

If $I_{2}^{\prime} \neq J_{2}$ (equivalently, if $I_{1}^{\prime} \neq J_{1}$ ), then we iterate the procedure, until eventually we replace the initial segments $I_{1}$ and $I_{2}$ with $J_{1}$ and $J_{2}$, respectively, and the assertion will then follow from (13). Thus, to complete the proof of i) it remains to establish (13). To this end, we have to distinguish two cases.

Suppose first that $\left|\partial^{*} I_{1}\right|>\left|\operatorname{cl}^{*}\left(I_{2}\right) \backslash I_{2}\right|$, so that $I_{2}$ is not closed in view of $\left|I_{1}\right| \leq\left|I_{2}\right|$; consequently, $\left|\partial I_{2}\right|=\left|\partial^{*} I_{2}\right|>0$ and $\operatorname{int}\left(I_{2}\right)=\operatorname{int}^{*}\left(I_{2}\right)$. In this case we have $\delta=$ $\left|\operatorname{cl}^{*}\left(I_{2}\right)\right|-\left|I_{2}\right|$ whence $\left|I_{2}^{\prime}\right|=\left|\operatorname{cl}^{*}\left(I_{2}\right)\right|$ and therefore $I_{2}^{\prime}=\operatorname{cl}^{*}\left(I_{2}\right)$ and $\operatorname{int}^{*}\left(I_{2}^{\prime}\right)=\operatorname{int}^{*}\left(I_{2}\right)$; also, $\left|I_{1}^{\prime}\right|>\left|I_{1}\right|-\left|\partial^{*} I_{1}\right|=\left|\operatorname{int}^{*}\left(I_{1}\right)\right|$, implying $\operatorname{int}^{*}\left(I_{1}^{\prime}\right)=\operatorname{int}^{*}\left(I_{1}\right)$. As a result, using (11), 
we get

$$
\begin{aligned}
\sigma_{n}\left(I_{1}\right)+\sigma_{n}\left(I_{2}\right) & =\sigma_{n}\left(\operatorname{int}^{*}\left(I_{1}\right)\right)+\sigma_{n-1}\left(\partial^{*} I_{1}\right)+\sigma_{n}\left(\operatorname{int}^{*}\left(I_{2}\right)\right)+\sigma_{n-1}\left(\partial^{*} I_{2}\right) \\
& =\sigma_{n}\left(\operatorname{int}^{*}\left(I_{1}^{\prime}\right)\right)+\sigma_{n}\left(\operatorname{int}^{*}\left(I_{2}^{\prime}\right)\right)+\left(\sigma_{n-1}\left(\partial^{*} I_{1}\right)+\sigma_{n-1}\left(\partial^{*} I_{2}\right)\right) .
\end{aligned}
$$

We now notice that $\partial^{*} I_{2}^{\prime}$ is a closed initial segment in $\mathbb{N}_{0}^{n-1}$, and that

$$
\left|\partial^{*} I_{1}^{\prime}\right|+\left|\partial^{*} I_{2}^{\prime}\right|=\left(\left|\partial^{*} I_{1}\right|-\delta\right)+\left(\left|\partial^{*} I_{2}\right|+\delta\right)=\left|\partial^{*} I_{1}\right|+\left|\partial^{*} I_{2}\right| .
$$

Also,

$$
\left|\partial^{*} I_{2}^{\prime}\right|=\left|I_{2}^{\prime}\right|-\left|\operatorname{int}^{*}\left(I_{2}^{\prime}\right)\right|=\left|\operatorname{cl}^{*}\left(I_{2}\right)\right|-\left|\operatorname{int}^{*}\left(I_{2}\right)\right|>\left|I_{2}\right|-\left|\operatorname{int}^{*}\left(I_{2}\right)\right|=\left|\partial^{*} I_{2}\right| .
$$

Therefore, an inductive application of i) in dimension $n-1$ yields

$$
\sigma_{n-1}\left(\partial^{*} I_{1}\right)+\sigma_{n-1}\left(\partial^{*} I_{2}\right) \geq \sigma_{n-1}\left(\partial^{*} I_{1}^{\prime}\right)+\sigma_{n-1}\left(\partial^{*} I_{2}^{\prime}\right) .
$$

Combining this with (14), and using (11) once again, we obtain

$$
\begin{aligned}
\sigma_{n}\left(I_{1}\right)+\sigma_{n}\left(I_{2}\right) & \geq \sigma_{n}\left(\operatorname{int}^{*}\left(I_{1}^{\prime}\right)\right)+\sigma_{n}\left(\operatorname{int}^{*}\left(I_{2}^{\prime}\right)\right)+\sigma_{n-1}\left(\partial^{*} I_{1}^{\prime}\right)+\sigma_{n-1}\left(\partial^{*} I_{2}^{\prime}\right) \\
& =\sigma_{n}\left(I_{1}^{\prime}\right)+\sigma_{n}\left(I_{2}^{\prime}\right)
\end{aligned}
$$

which is the desired estimate (13).

Now suppose that $\left|\partial^{*} I_{1}\right| \leq\left|\mathrm{cl}^{*}\left(I_{2}\right) \backslash I_{2}\right|$. In this case $\delta=\left|\partial^{*} I_{1}\right|, I_{1}^{\prime}=$ int $^{*}\left(I_{1}\right)$, $\operatorname{int}^{*}\left(I_{2}^{\prime}\right)=\operatorname{int}\left(I_{2}\right)$, and

$$
\left|\partial^{*} I_{2}^{\prime}\right|=\left|I_{2}^{\prime} \backslash \operatorname{int}\left(I_{2}\right)\right|=\left|\partial I_{2}\right|+\delta=\left|\partial^{*} I_{1}\right|+\left|\partial I_{2}\right| .
$$

There are two further sub-cases.

If $I_{2}$ is closed, then (15) gives $\left|\partial^{*} I_{2}^{\prime}\right|=\left|\partial^{*} I_{1}\right|$; as a result, using (11) we get

$$
\begin{aligned}
\sigma_{n}\left(I_{1}\right)+\sigma_{n}\left(I_{2}\right) & \geq\left(\sigma_{n}\left(\operatorname{int}^{*}\left(I_{1}\right)\right)+\sigma_{n-1}\left(\partial^{*} I_{1}\right)\right)+\sigma_{n}\left(I_{2}\right) \\
& =\sigma_{n}\left(I_{1}^{\prime}\right)+\left(\sigma_{n-1}\left(\partial^{*} I_{1}\right)+\sigma_{n}\left(\operatorname{int}\left(I_{2}\right)\right)\right) \\
& =\sigma_{n}\left(I_{1}^{\prime}\right)+\left(\sigma_{n-1}\left(\partial^{*} I_{2}^{\prime}\right)+\sigma_{n}\left(\operatorname{int}^{*}\left(I_{2}^{\prime}\right)\right)\right) \\
& =\sigma_{n}\left(I_{1}^{\prime}\right)+\sigma_{n}\left(I_{2}^{\prime}\right),
\end{aligned}
$$

which is (13). (The inequality in (16) is strict if and only if $I_{1}$ is a singleton; this fact will be used in the forthcoming proof of ii).)

If $I_{2}$ is not closed, then $\partial I_{2}=\partial^{*} I_{2}$ and $\operatorname{int}^{*}\left(I_{2}\right)=\operatorname{int}\left(I_{2}\right)=\operatorname{int}^{*}\left(I_{2}^{\prime}\right)$. Recalling (15) , in this case we apply ii) inductively in dimension $n-1$ to get

$$
\sigma_{n-1}\left(\partial^{*} I_{1}\right)+\sigma_{n-1}\left(\partial^{*} I_{2}\right)>\sigma_{n-1}\left(\partial^{*} I_{2}^{\prime}\right)
$$

whence, by (11),

$$
\begin{aligned}
\sigma_{n}\left(I_{1}\right)+\sigma_{n}\left(I_{2}\right) & \geq \sigma_{n}\left(\operatorname{int}^{*}\left(I_{1}\right)\right)+\left(\sigma_{n}\left(\operatorname{int}^{*}\left(I_{2}\right)\right)+\sigma_{n-1}\left(\partial^{*} I_{1}\right)+\sigma_{n-1}\left(\partial^{*} I_{2}\right)\right) \\
& >\sigma_{n}\left(I_{1}^{\prime}\right)+\left(\sigma_{n}\left(\operatorname{int}^{*}\left(I_{2}^{\prime}\right)\right)+\sigma_{n-1}\left(\partial^{*} I_{2}^{\prime}\right)\right) \\
& =\sigma_{n}\left(I_{1}^{\prime}\right)+\sigma_{n}\left(I_{2}^{\prime}\right) .
\end{aligned}
$$


This establishes (13), and therefore i).

Finally, we prove ii). Without loss of generality, we assume $\left|I_{1}\right| \leq\left|I_{2}\right|$. If

$$
\left|\mathrm{cl}^{*}\left(I_{2}\right) \backslash I_{2}\right| \leq\left|I_{1}\right|
$$

then we re-use the above argument for i) with $J_{2}:=\mathrm{cl}^{*}\left(I_{2}\right)$ and $J_{1}:=\mathcal{I}_{n}\left(\left|I_{1}\right|+\left|I_{2}\right|-\left|J_{2}\right|\right)$, defining $\delta$ by (12) and then letting $I_{1}^{\prime}:=\mathcal{I}_{n}\left(\left|I_{1}\right|-\delta\right)$ and $I_{2}^{\prime}:=\mathcal{I}_{n}\left(\left|I_{2}\right|+\delta\right)$, to have the estimate (13). We notice that if $\left|I_{1}\right|>1$, then $\delta \leq\left|\partial^{*} I_{1}\right|<\left|I_{1}\right|$, implying $\left|I_{1}^{\prime}\right| \geq 1$; moreover, if $\left|I_{1}\right|=1$, then the inequality in (13) is strict, as it follows from (17) and the remark following (16). (This reflects the geometrically obvious fact that if $I_{1}$ consists of one single point, then moving this point to $I_{2}$ reduces the total sum of the hyperplane projections by at least 1.)

Continuing in this way, we find initial segments $I_{1}^{\prime \prime} \subseteq I_{2}^{\prime \prime}$ satisfying $\left|I_{1}^{\prime \prime}\right|+\left|I_{2}^{\prime \prime}\right|=\left|I_{1}\right|+\left|I_{2}\right|$ and

$$
\sigma_{n}\left(I_{1}^{\prime \prime}\right)+\sigma_{n}\left(I_{2}^{\prime \prime}\right) \leq \sigma_{n}\left(I_{1}\right)+\sigma_{n}\left(I_{2}\right)
$$

such that either $I_{1}^{\prime \prime}=\varnothing, I_{2}^{\prime \prime}=I$, and (19) holds actually as a strict inequality, or $I_{1}^{\prime \prime} \neq \varnothing$ and $\left|\mathrm{cl}^{*}\left(I_{2}^{\prime \prime}\right) \backslash I_{2}^{\prime \prime}\right|>\left|I_{1}^{\prime \prime}\right|$, cf. (18). In the former case ii) follows readily. In the latter case, recalling that $I=\mathcal{I}_{n}\left(\left|I_{1}^{\prime \prime}\right|+\left|I_{2}^{\prime \prime}\right|\right)$, we have $\operatorname{int}^{*}(I)=\operatorname{int}\left(I_{2}^{\prime \prime}\right)$, whence

$$
\left|\partial^{*} I\right|=\left(\left|I_{1}^{\prime \prime}\right|+\left|I_{2}^{\prime \prime}\right|\right)-\left|\operatorname{int}\left(I_{2}^{\prime \prime}\right)\right|=\left|\partial I_{2}^{\prime \prime}\right|+\left|I_{1}^{\prime \prime}\right| ;
$$

consequently, using (11), and then applying ii) inductively,

$$
\begin{aligned}
\sigma_{n}(I) & =\sigma_{n}\left(\operatorname{int}^{*}(I)\right)+\sigma_{n-1}\left(\partial^{*} I\right) \\
& =\sigma_{n}\left(\operatorname{int}\left(I_{2}^{\prime \prime}\right)\right)+\sigma_{n-1}\left(\left|\partial I_{2}^{\prime \prime}\right|+\left|I_{1}^{\prime \prime}\right|\right) \\
& \leq \sigma_{n}\left(\operatorname{int}\left(I_{2}^{\prime \prime}\right)\right)+\sigma_{n-1}\left(\partial I_{2}^{\prime \prime}\right)+\sigma_{n-1}\left(I_{1}^{\prime \prime}\right) .
\end{aligned}
$$

However, as a version of (11) (essentially equivalent to (9)), we have

$$
\sigma_{n}\left(\operatorname{int}\left(I_{2}^{\prime \prime}\right)\right)+\sigma_{n-1}\left(\partial I_{2}^{\prime \prime}\right)=\sigma_{n}\left(I_{2}^{\prime \prime}\right),
$$

and by iii),

$$
\sigma_{n-1}\left(I_{1}^{\prime \prime}\right)<\sigma_{n}\left(I_{1}^{\prime \prime}\right) .
$$

Combining (20)-(22) and (19), we get

$$
\sigma_{n}(I)<\sigma_{n}\left(I_{1}^{\prime \prime}\right)+\sigma_{n}\left(I_{2}^{\prime \prime}\right) \leq \sigma_{n}\left(I_{1}\right)+\sigma_{n}\left(I_{2}\right) .
$$

This completes the proof of ii).

\section{Proof of Theorem 1}

As explained in the introduction, it suffices to show that for any finite set $A \subset \mathbb{N}_{0}^{n}$, writing $m:=|A|$, we have $\sigma_{n}(A) \geq \sigma_{n}(m)$. The proof goes by induction on $n$, the base case $n=1$ being trivial as the zero-dimensional projection of any nonempty set has by convention, size 1 . The assertion is readily verified for $m \in\{0,1\}$, too. Suppose thus that $\min \{n, m\} \geq 2$. 
Our strategy is to start out with any minimiser $A \subset \mathbb{N}_{0}^{n}$ and modify it, in a finite number of rearrangements not increasing the projection sum, to get the initial segment $\mathcal{I}_{n}(|A|)$. We achieve this in several steps, some of which may need to be iterated, as explained below.

Step 1. Let $H$ (for "height") denote this number of non-empty slabs of $A$. We permute the slabs so that the number of elements of $A$ in any higher slab does not exceed the number of elements in a lower slab; that is, letting $A^{(k)}:=A \cap\left(k e_{n}+\mathcal{L}_{n}\right)$, we have $\left|A^{(0)}\right| \geq \cdots \geq\left|A^{(H-1)}\right|>0$ and $\left|A^{(k)}\right|=0$ for $k \notin[0, H-1]$. This does not affect the projection sum $\sigma_{n}(A)$ as each non-horizontal projection of $A$ is the disjoint union of the corresponding projections of the slabs:

$$
\pi_{i}(A)=\bigcup_{k \geq 0} \pi_{i}\left(A^{(k)}\right), \quad i \in[1, n-1] .
$$

To simplify the notation, we keep denoting by $A$ the set under consideration. Clearly, the number of non-empty slabs of $A$ remains equal to $H$.

We now replace each slab $A^{(k)}$ with the initial segment $\mathcal{I}_{n-1}\left(\left|A^{(k)}\right|\right)$, without enlarging the projection sum. (It is readily seen that the horizontal projection does not increase, and the sum of the side projections does not increase in view of (23) and by the induction hypothesis.) We use the same notation $A$ for the new set obtained in this way, but from now on we assume that each slab of $A$ is an $(n-1)$-dimensional initial segment. Since the sequence $\left(\left|A^{(k)}\right|\right)_{k \geq 0}$ is non-increasing, this implies $A^{(k+1)} \subseteq e_{n}+A^{(k)}$ for each $k \geq 0$.

Let $K$ denote the largest edge size of the closed $(n-1)$-dimensional initial segment $\operatorname{cl}\left(A^{(0)}\right)$; that is, the size of the projection of $A^{(0)}$ onto the coordinate axis $\mathcal{X}_{1}$. If $K<H$, then we swap the coordinate axes $\mathcal{X}_{1}$ and $\mathcal{X}_{n}$ so that the number of non-empty slabs decreases to $K$, and repeat the whole procedure.

We keep permuting the slabs and swapping the axes until $A$ gets rearranged so that the number $H$ of non-empty slabs does not exceed the largest edge size $K$ of the closure $\operatorname{cl}\left(A^{(0)}\right)$ of the lowest slab, and each slab is an $(n-1)$-dimensional initial segment.

Step 2. A repeated application of this step will ensure that all non-empty slabs of $A$, with the possible exception of the highest slab $A^{(H-1)}$, have the same interior. Assuming this is not the case, there are integers $k \in[1, H-2]$ with $\left|A^{(k)}\right|<\left|\operatorname{int}\left(A^{(0)}\right)\right|$. Let $k$ be the smallest such integer. If, indeed, we had $\left|A^{(k)}\right|+\left|A^{(H-1)}\right| \leq\left|\operatorname{int}\left(A^{(0)}\right)\right|$, then we would be able to remove $A^{(H-1)}$ from $A$ and replace $A^{(k)}$ with the (appropriate vertical translate of the) initial segment $\mathcal{I}_{n-1}\left(\left|A^{(k)}\right|+\left|A^{(H-1)}\right|\right)$, without changing the horizontal projection $\pi_{n}(A)$. By Lemma 1 ii), this would result in the strict decrease of the sum of the non-horizonal projections, contradicting the assumption that $A$ is a minimiser. Thus, we have $\left|A^{(k)}\right|+\left|A^{(H-1)}\right|>\left|\operatorname{int}\left(A^{(0)}\right)\right|$, and we replace the slab $A^{(k)}$ with $\operatorname{int}\left(A^{(0)}\right)$, and the upper slab $A^{(H-1)}$ with the initial segment $\mathcal{I}_{n-1}\left(\left|A^{(H-1)}\right|+\left|A^{(k)}\right|-\left|\operatorname{int}\left(A^{(0)}\right)\right|\right)$; by Lemma 1 i), applied with $I_{1}=A^{(H-1)}, I_{2}=A^{(k)}$, and $J_{2}=\operatorname{int}\left(A^{(0)}\right)$, this does not 
increase the sum of non-horizontal projections of $A$, and it is clear that the horizontal projection $\pi_{n}(A)$ remains unchanged.

We emphasize that Step 2 affects neither the number $H$ of the slabs of $A$, nor the lower slab $A^{(0)}$, and that if this step ever gets applied, then the resulting set satisfies $\left|A^{(0)}\right|>\left|A^{(H-1)}\right|$.

Repeating Step 2, we ensure that all non-empty slabs of $A$, excepting perhaps $A^{(H-1)}$, have their interiors identical to that of $A^{(0)}$, which we assume to hold from now on.

Step 3. Recall that by $K$ we denote the largest edge size of the closed $(n-1)$-dimensional segment $\operatorname{cl}\left(A^{(0)}\right)$. As a result of the rearrangements of Step 1, we have $K \geq H$, and the present Step 3 is to be repeated as long as the strict inequality $K>H$ holds, or until $A$ gets rearranged as in initial segment.

If $A$ can be cast as an initial segment just by relabelling the coordinate axes, this will complete the proof; this scenario will be referred to as a trivial exit. Otherwise, we are going to cut from $A$ a "vertical slab" resting on the strict boundary $\partial^{*} A^{(0)}$, and place it as a new horizontal slab (as a result of which $H$ will grow by 1 ). Given that only "side" projections of both slabs contribute to the projection sum $\sigma_{n}(A)$, it will not be affected by this rearrangement. Formally, we need to consider two cases.

The first case is $\left|A^{(H-1)}\right| \geq \mid$ int $^{*}\left(A^{(0)}\right) \mid$ (covering, in particular, the situation where $H=1)$. In this case each slab of $A$ contains a vertical translate of the strict interior $\operatorname{int}^{*}\left(A^{(0)}\right)$, and we define $A^{\prime}$ to be the union of all these $H$ translates, and let $A^{\prime \prime}:=$ $A \backslash A^{\prime}$. Notice that $A^{\prime \prime}$ is a non-empty subset of a hyperplane parallel to one of the (non-horizontal) coordinate hyperplanes. Considering $A^{\prime \prime}$ as an $(n-1)$-dimensional set, we have

$$
\sigma_{n}(A)=\sigma_{n}\left(A^{\prime}\right)+\sigma_{n-1}\left(A^{\prime \prime}\right)
$$

Observe that if we replace the set $A^{\prime \prime}$ with the initial segment $\mathcal{I}_{n-1}\left(\left|A^{\prime \prime}\right|\right)$, by the induction assumption and the assumption that $A$ is a minimiser, this will not affect the projection sum $\sigma_{n-1}\left(A^{\prime \prime}\right)$.

If $H=K-1$, then $A^{\prime}$ is the union of $K-1$ vertical translates of the closed initial segment $\operatorname{int}^{*}\left(A^{(0)}\right)$, which is the $n$-dimensional axes-aligned rectangular parallelepiped with the edge sizes $K-1$ and (possibly) $K$. The set $A^{\prime \prime}$ is strictly smaller than the face of $A^{\prime}$ it is attached to, for otherwise the original set $A$ would be a closed initial segment, up to relabelling of the coordinate axes, and we would have the trivial exit scenario. Hence $A^{\prime \prime}$ can be replaced with the initial segment $\mathcal{I}_{n-1}\left(\left|A^{\prime \prime}\right|\right)$ and re-attached to the appropriate face of $A^{\prime}$ to get a set which, up to a coordinate axes relabelling, is an initial segment, completing the proof (for the present subcase $H=K-1$ ). Observe, for the forthcoming proof of Theorem 2 , that the fact that $A^{\prime \prime}$ is strictly smaller than the face of $A^{\prime}$ it is attached to, precludes the output initial segment $\mathcal{I}_{n}(|A|)$ from being closed. 
Assuming now that $H<K-1$, let $K_{1} \geq \cdots \geq K_{n-1}$ be the edge sizes of int ${ }^{*}\left(A^{(0)}\right)$, so that $K \geq K_{1}$ and $K_{n-1} \geq K-1$. We have then

$$
\left|A^{\prime \prime}\right| \leq\left|\partial^{*} A^{(0)}\right| \cdot H<K_{1} \cdots K_{n-2} \cdot(K-1) \leq K_{1} \cdots K_{n-2} \cdot K_{n-1}=\left|\operatorname{int}^{*}\left(A^{(0)}\right)\right| .
$$

It follows that we can $\operatorname{detach} A^{\prime \prime}$ from $A$, replace it with the initial segment $\mathcal{I}_{n-1}\left(\left|A^{\prime \prime}\right|\right)$, and re-attach as the upper slab, thus increasing $H$ by 1 and changing the lower slab of $A$ from the original $A^{(0)}$ to its strict interior int ${ }^{*}\left(A^{(0)}\right)$. Notice that, as a consequence of (25), the resulting set has fewer elements in its upper slab than in the lower slab.

For the second case $\left|A^{(H-1)}\right|<\left|\operatorname{int}^{*}\left(A^{(0)}\right)\right|$, we define $A^{\prime}$ to be the union of $H-1$ (rather than $H$ as in the first case) vertical translates of the set $\operatorname{int}^{*}\left(A^{(0)}\right)$, with the coordinate $\mathcal{X}_{n}$ ranging from 0 to $H-2$, and we let $A^{\prime \prime}:=A \backslash\left(A^{\prime} \cup A^{(H-1)}\right)$. Thus $A^{\prime}$ is an axes-aligned rectangular parallelepiped, attached to two faces of which are the $(n-1)$-dimensional sets $A^{\prime \prime}$ and $A^{(H-1)}$; hence,

$$
\sigma_{n}(A)=\sigma_{n}\left(A^{\prime}\right)+\sigma_{n-1}\left(A^{\prime \prime}\right)+\sigma_{n-1}\left(A^{(H-1)}\right) .
$$

We now rearrange $A^{\prime \prime}$ as an $(n-1)$-dimensional initial segment, and then detach it from $A$ and re-attach as either the upper, or the second-from-the-top slab, to retain the nonincreasing order of slab sizes. As above, this rearrangement makes $H$ larger by 1 , and changes the lower slab of $A$ from the original $A^{(0)}$ to its strict interior int ${ }^{*}\left(A^{(0)}\right)$. Also, keeping denoting the notation for the edges of int ${ }^{*}\left(A^{(0)}\right)$, similarly to (25) we have

$$
\left|A^{\prime \prime}\right| \leq\left|\partial^{*} A^{(0)}\right| \cdot(H-1) \leq K_{1} \cdots K_{n-2} \cdot(H-1)<K_{1} \cdots K_{n-2} \cdot K_{n-1}=\left|\operatorname{int}^{*}\left(A^{(0)}\right)\right|
$$

thus, as above, the size of the horizontal projection of $A$ becomes $\mid$ int $^{*}\left(A^{(0)}\right) \mid$.

Finally (just for the second-from-the-top slab) we invoke the rearrangement of Step 2 to ensure that all, but the upper slab of $A$ have the same interior; hence, are actually identical closed initial segments since $A^{(0)}$ is a closed initial segment.

Observe that, unless we have achieved our goal of rearranging $A$ as an initial segment (as in the trivial exit scenario or the case where $\left|A^{(H-1)}\right| \geq\left|\operatorname{int}^{*}\left(A^{(0)}\right)\right|$ and $H=K-1$ ), the procedure introduced in Step 3 results in $H$ growing by 1 , with the strict inequality $\left|A^{(H-1)}\right|<\left|A^{(0)}\right|$ for the rearranged $A$, and with $A^{(0)}$ being a closed $(n-1)$-dimensional initial segment. In addition, the parameter $K$ is either unchanged, or decreases by 1 , the latter happening if and only if the new "base slab" $A^{(0)}$ is an $(n-1)$-dimensional cube. Therefore, if the new parameters satisfy $K<H$ (that is, $H=K+1$ ), then the set $A$ got rearranged into a cube with the edge size $K$, with an $(n-1)$-dimensional initial segment attached to its upper face as boundary; that is, into a (non-closed) $n$-dimensional initial segment.

We have shown that, applying Step 3 repeatedly, we will either rearrange $A$ as in initial segment, or arrive in the special situation where $K=H$, dealt with at Step 4 below. 
Step 4. For this last step of our algorithm we assume that $K=H$ where, we recall, $H$ is the number of non-empty slabs of $A$, and $K$ is the largest size of a projection of the lower slab $A^{(0)}$ onto a non-vertical coordinate axis. This step is not to be iterated; it is applied at most once and after completing it, $A$ will be rearranged as an initial segment.

If $A$ can be rearranged as an initial segment by merely relabelling the coordinate axes, then the algorithm stops and proof is completed; as in Step 3, this situation will be referred to as the trivial exit. Assuming that we have not exited trivially, as in the above Step 3, we consider two cases.

The first one is a straightforward modification of the corresponding case of Step 3 , with $\operatorname{int}^{*}\left(A^{(0)}\right)$ replaced by $\operatorname{int}\left(A^{(0)}\right)$ (and the equality $H=K-1$ replaced by $H=K$ ). Namely, if $\left|A^{(H-1)}\right| \geq\left|\operatorname{int}\left(A^{(0)}\right)\right|$, then we define $A^{\prime}$ to be the union of $H=K$ vertical translates of $\operatorname{int}\left(A^{(0)}\right)$, one on top of the other, and let $A^{\prime \prime}=A \backslash A^{\prime}$. The set $A^{\prime}$ is an $n$-dimensional axes-aligned rectangular parallelepiped with the maximum edge size $K$ and minimum edge size at least $K-1$, and $A^{\prime \prime}$ is attached to a maximal-sized face of $A^{\prime}$. Hence, having $A^{\prime \prime}$ replaced by the $(n-1)$-dimensional initial segment $\mathcal{I}_{n-1}\left(\left|A^{\prime \prime}\right|\right)$, the set $A$ can be cast as the initial segment by relabelling the coordinate axes.

Preparing the ground for the proof of Theorem 2 in the next section, we notice that $A^{\prime \prime}$ is non-empty, and is strictly smaller than the face of $A^{\prime}$ it is attached to, for otherwise $A$ can be rearranged as an initial segment by relabelling the coordinate axes, which would lead to the trivial exit scenario. This precludes the output set $\mathcal{I}_{n}(|A|)$ from being closed.

Moving on to the second case, for the rest of Step 4 we assume that $\left|A^{(H-1)}\right|<$ $\left|\operatorname{int}\left(A^{(0)}\right)\right|$. The set $A \backslash A^{(H-1)}$ consists of $H-1=K-1$ slabs, each one being an $(n-1)$-dimensional initial segment with the same interior $\operatorname{int}\left(A^{(0)}\right)$. All projections onto the non-vertical axes of the closed $(n-1)$-dimensional initial segment $\operatorname{int}\left(A^{(0)}\right)$ have size $K$ or $K-1$; therefore, the stack of $K-1$ vertical translates of $\operatorname{int}\left(A^{(0)}\right)$, which we denote $A^{\prime}$, is a closed $n$-dimensional initial segment. Furthermore, the set $A^{\prime \prime}:=A \backslash\left(A^{\prime} \cup A^{(H-1)}\right)$ is nonempty, for otherwise $A$ could be rearranged as the initial segment by relabelling the coordinate axes, which is ruled out by trivial exit scenario.

Thus, attached to the upper horizontal face of $A^{\prime}$ is the slab $A^{(H-1)}$, and to some of its "vertical" faces - the "vertical slab" $A^{\prime \prime}$ (which, by the construction, is strictly smaller than the face of $A^{\prime}$ it is attached to). Our plan is to replace $A^{\prime \prime}$ by the same-sized $(n-1)$-dimensional initial segment, and then apply Lemma 1 to transfer elements from $A^{(H-1)}$ and $A^{\prime \prime}$ to $A^{\prime}$, to augment this latter set to its strict closure $\mathrm{cl}^{*}\left(A^{\prime}\right)$.

Since the horizontal projections of $A^{(H-1)}$ and $A^{\prime \prime}$ are disjoint, we have

$$
\sigma_{n}(A)=\sigma_{n}\left(A^{\prime}\right)+\sigma_{n-1}\left(A^{\prime \prime}\right)+\sigma_{n-1}\left(A^{(H-1)}\right) .
$$

By the induction assumption, replacing $A^{\prime \prime}$ with the initial segment $\mathcal{I}_{n-1}\left(\left|A^{\prime \prime}\right|\right)$ does not increase the summand $\sigma_{n-1}\left(A^{\prime \prime}\right)$. As usual, we do not change the notation, but assume below that $A^{\prime \prime}$ is an $(n-1)$-dimensional initial segment. 
Let $I_{1}$ and $I_{2}$ be the smallest and the largest among the initial segments $A^{\prime \prime}$ and $A^{(H-1)}$, respectively, and let $J_{2}:=\mathrm{cl}^{*}\left(A^{\prime}\right) \backslash A^{\prime}$; thus, $J_{2}$ is the face to be added to the closed initial segment $A^{\prime}$ in order to obtain the "next" closed initial segment $\mathrm{cl}^{*}\left(A^{\prime}\right)$. Notice that, by the virtue of the cube order, $\left|J_{2}\right|$ is the size of the largest face of $A^{\prime}$, whence $\left|J_{2}\right|>\left|I_{2}\right|$. Also notice that $I_{1}$ and $I_{2}$ are non-empty as so are $A^{\prime \prime}$ and $A^{(H-1)}$. If we had $\left|J_{2}\right| \geq\left|I_{1}\right|+\left|I_{2}\right|$ then, applying Lemma 10ii), we could have replaced $A^{\prime \prime}$ and $A^{(H-1)}$ with one single $(n-1)$-dimensional initial segment of size $\left|A^{\prime \prime}\right|+\left|A^{(H-1)}\right|$ attached to the largest face of $A$, decreasing the sum of the projections of $A$; this would contradict the assumptions that $A$ is a minimiser. Therefore we have $\left|I_{1}\right| \leq\left|I_{2}\right| \leq\left|J_{2}\right|<\left|I_{1}\right|+\left|I_{2}\right|$, and we set $J_{1}:=\mathcal{I}_{n-1}\left(\left|I_{1}\right|+\left|I_{2}\right|-\left|J_{2}\right|\right)$ and replace $A^{\prime \prime}$ and $A^{(H-1)}$ with the initial segments $J_{2}$ attached to the appropriate face of $A^{\prime}$ to convert it to $\mathrm{cl}^{*}\left(A^{\prime}\right)$, and $J_{1}$ attached as a boundary to $\mathrm{cl}^{*}\left(A^{\prime}\right)$ (which can be done in view of $\left|J_{1}\right| \leq\left|J_{2}\right|$ ). This rearranges $A$ as an initial segment. Observe that for this last exit scenario, the output set $\mathcal{I}_{n}(|A|)$ cannot be closed in view of the estimate $0<\left|J_{1}\right|<\left|J_{2}\right|$ resulting from

$$
\left|J_{1}\right|=\left|I_{1}\right|+\left|I_{2}\right|-\left|J_{2}\right| \leq 2\left|I_{2}\right|-\left|J_{2}\right|<\left|J_{2}\right|
$$

\section{Proof of Theorem 2}

Applying the argument presented after the statement of Theorem 1 in Section 2, we assume without loss of generality that $A \subset \mathbb{N}_{0}^{n}$.

We define similarities to be bijective transformations of the set $\mathbb{Z}^{n}$ involving (finitely many) permutations of the horizontal hyperplanes, axes relabellings, and compositions thereof. Thus, two sets in $\mathbb{Z}^{n}$ are similar if they can be obtained from each other by a finite series of permutations of the slabs and relabellings of the coordinate axes. For $n=1$, any two sets of the same size are similar, proving the assertion in this case. For $n \geq 1$, if $A_{1}, A_{2} \subset \mathbb{Z}^{n}$ are similar, finite sets, then $\sigma_{n}\left(A_{1}\right)=\sigma_{n}\left(A_{2}\right)$.

Our argument uses induction by $n$ and is based on a careful examination of the proof of Theorem 1 in the previous section; in fact, it has been prepared by the observations made there, and particularly at the key Steps 3 and 4. Clearly, it suffices to show that if $A \subset \mathbb{N}_{0}^{n}$ is a minimiser such that $\mathcal{I}_{n}(|A|)$ is closed, then all rearrangements made in the course of the proof are, in fact, similarities; that is, involve only permuting the slabs and relabelling the coordinates.

Suppose thus that $A \subseteq \mathbb{N}_{0}^{n}$ is a minimiser with $\mathcal{I}_{n}(|A|)$ closed. Inspecting the proof of Theorem 1, we make the two following observations.

(i) When Step 3 is applied with a non-trivial exit, the output set has strictly fewer elements in its upper slab than in the lower one; in particular, this set cannot be converted into a closed initial segment by relabelling the axes. 
(ii) The only way that Steps 3 and 4 can yield a closed initial segment is that they are exited trivially; in particular, the input set must be an axes-aligned rectangular parallelepiped.

It follows that after completing Steps 1 and 2 , the set $A$ may have only required an axes relabelling to get transformed into a closed initial segment. In fact, no application of Step 2 would have been possible either, for any such application results in a set with its upper slab strictly smaller than the lower one.

Thus, rearranging $A$ to $\mathcal{I}_{n}(|A|)$ has only required Step 1 followed, possibly, by an axes relabelling. We recall that Step 1 consists of a number of iterations of the procedure that involves permuting slabs, replacing each slab with the equal-sized $(n-1)$-dimensional initial segment, and swapping the axes.

Consider the last iteration of Step 1; specifically, the middle part of the iteration replacing each slab with the same-sized $(n-1)$-dimensional initial segment. Let $A^{\prime}$ and $A^{\prime \prime}$ denote the corresponding input and output sets. Thus, $A^{\prime \prime}$ is an axes-aligned rectangular parallelepiped, with all of its edges differing in size by 1 at most. It follows that the common size of all slabs of $A^{\prime \prime}$ is the cardinality of a closed $(n-1)$-dimensional initial segment, and we invoke the induction hypothesis to conclude that on the last iteration, replacing each slab of $A^{\prime}$ with an $(n-1)$-dimensional initial segment is induced by an $(n-1)$-dimensional similarity transformation.

On the other hand, we note that the horizontal projection of $A^{\prime}$ is the union of its slabs, viewed as $(n-1)$-dimensional sets (which have the same common size, as so do the slabs of $A^{\prime \prime}$ ). Since we are working with minimisers, this implies that the size of this union is equal to the size of each individual slab of $A^{\prime}$, and therefore all the slabs of $A^{\prime}$ are actually identical. As a result, the same similarity transformation that converts, say, the lower slab of $A^{\prime}$ into a closed $(n-1)$-dimensional initial segment, will also work for all other slabs of $A^{\prime}$ converting them into (identical) closed initial segments. Extending this transformation to act as an identity on the last coordinate, we obtain an $n$-dimensional similarity transformation that replaces all slabs of $A^{\prime}$ with the $(n-1)$-dimensional initial segments.

We conclude that rearranging $A^{\prime}$ into $A^{\prime \prime}$, and hence the whole last iteration of Step 1, can be achieved using a similarity transformation. Making our way backwards, the same is true for all the preceding iterations. Consequently, the whole Step 1 acted on $A$ as a similarity, and the assertion follows.

\section{Proof of the equivalence of Theorems 1 and 3}

Given integers $n, s \geq 1$ and $m_{1}, \ldots, m_{s} \geq 0$, consider the set $A \subset \mathbb{N}_{0}^{n}$ with $s$ non-empty slabs such that for every $k \in[1, s]$, the $k$ th slab is the $(n-1)$-dimensional initial segment of length $m_{k}$. Since the side projections of all these slabs are pairwise disjoint, while the 
horizontal projections are all contained in the largest of them, we have

$$
\sigma_{n}(A)=\sigma_{n-1}\left(m_{1}\right)+\cdots+\sigma_{n-1}\left(m_{s}\right)+\max \left\{m_{1}, \ldots, m_{s}\right\} .
$$

However, the left-hand side is at least as large as $\sigma_{n}(|A|)=\sigma_{n}\left(m_{1}+\cdots+m_{s}\right)$ by Theorem 1, Hence Theorem 1 implies Theorem 3 ,

Conversely, assuming Theorem [3, one can prove Theorem 1 by induction on $n$, as follows. Given a finite set $A \subset \mathbb{N}_{0}^{n}$, consider the slab decomposition $A=A^{(1)} \cup \cdots \cup A^{(s)}$, with the $A^{(k)}$ listing all non-empty slabs of $A$. For each $k \in[1, s]$, let $m_{k}:=\left|A^{(k)}\right|$. Trivially, we have $\left|\pi_{n}(A)\right| \geq \max \left\{m_{1}, \ldots, m_{s}\right\}$. Also, disjointness of the side projections yields

$$
\sum_{i=1}^{n-1}\left|\pi_{i}(A)\right|=\sum_{k=1}^{s} \sum_{i=1}^{n-1}\left|\pi_{i}\left(A^{(k)}\right)\right| .
$$

By the induction hypothesis, the double sum in the right-hand side is at least as large as $\sigma_{n-1}\left(m_{1}\right)+\cdots+\sigma_{n-1}\left(m_{s}\right)$. Therefore, by Theorem 3 ,

$$
\begin{aligned}
\sum_{i=1}^{n}\left|\pi_{i}(A)\right| \geq \sigma_{n-1}\left(m_{1}\right)+\cdots+\sigma_{n-1}\left(m_{s}\right)+\max \left\{m_{1}, \ldots, m_{s}\right\} & \\
& \geq \sigma_{n}\left(m_{1}+\cdots+m_{s}\right)=\sigma_{n}(|A|),
\end{aligned}
$$

as claimed by Theorem 1.

\section{APPENDIX: OnE-DIMENSIONAL PROJECTIONS}

It would be interesting to extend our results onto $k$-dimensional projections for all integers $k \in[1, n-2]$. Below we consider the case $k=1$, establishing the analogs of Theorems 1, 2, and 3 for the one-dimensional projections. In particular, we show that the sum of the sizes of these projections is also minimised on the initial segments of $\mathbb{N}_{0}^{n}$ with respect to the cube order, and prove a corresponding stability result.

For each $i \in[1, n]$, denote by $\rho_{i}$ the orthogonal projections of $\mathbb{R}^{n}$ onto the coordinate axis $\mathcal{X}_{i}$, and given a finite set $A \subset \mathbb{R}^{n}$, let

$$
\lambda_{n}(A):=\sum_{i=1}^{n}\left|\rho_{i}(A)\right| ;
$$

also, for integer $m \geq 0$ let $\lambda_{n}(m):=\lambda_{n}\left(\mathcal{I}_{n}(m)\right)$. Thus, for instance, $\lambda_{1}(m)=m$, $\lambda_{2}(m)=\sigma_{2}(m)$, and if $|A|=K^{n}$ with an integer $K \geq 1$, then by the arithmetic-geometric mean inequality,

$$
\lambda_{n}(A) \geq n\left(\prod_{i=1}^{n}\left|\rho_{i}(A)\right|\right)^{1 / n} \geq n|A|^{1 / n}=n K,
$$

with equality attained for the discrete cube $A=[0, K-1]^{n}$. 
The key to understanding the quantity $\lambda_{n}$ is the equality

$$
\lambda_{n}(m+1)= \begin{cases}\lambda_{n}(m)+1 & \text { if } \mathcal{I}_{n}(m) \text { is closed } \\ \lambda_{n}(m) & \text { otherwise }\end{cases}
$$

An immediate corollary is that if $K^{n} \leq m \leq(K+1)^{n}$, with a positive integer $K$, and $i \in[0, n]$ is the smallest integer such that $m \leq(K+1)^{i} K^{n-i}$, then

$$
\lambda_{n}(m)=\lambda_{n}\left((K+1)^{i} K^{n-i}\right)=n K+i,
$$

cf. (9).

As an analog of Theorems 1 and 2, we now have

Theorem 4. For every integer $n \geq 1$ and every finite set $A \subset \mathbb{R}^{n}$, letting $m:=|A|$, we have $\lambda_{n}(A) \geq \lambda_{n}(m)$. Moreover, if $m=(K+1)^{i} K^{n-i}$ with integers $K \geq 1$ and $i \in[0, n]$, then equality is attained if and only if $A$ is the Cartesian product of $i$ real sets of size $K+1$, and $n-i$ real sets of size $K$.

Although it is possible to prove Theorem 4 modifying the proofs of Theorems 1 and 2 to apply in our present settings, somewhat surprisingly, one can get away with a much easier, non-inductive argument.

Proof of Theorem 4. The case where $m=0$ is trivial, and we assume that $m>0$; that is, $A$ is non-empty. For each $j \in[1, n]$, let $m_{j}:=\left|\rho_{j}(m)\right|$; thus, $m \leq m_{1} \cdots m_{n}$ and $\lambda_{n}(A)=m_{1}+\cdots+m_{n}$. If the largest of the numbers $m_{j}$ exceeds the smallest of them by at least 2 , then we decrease by 1 the largest, and simultaneously increase by 1 the smallest; clearly, this operation does not affect the sum of the numbers, and their product will only get larger. Iterating, we will eventually find $n$ positive integers, the largest of them exceeding the smallest one by at most 1 , so that their product is at least $m$, and their sum is $\lambda_{n}(A)$. Denoting by $I$ the closed initial segment whose edges are determined by these resulting integers, we then have $m \leq|I|$ and $\lambda_{n}(A)=\lambda_{n}(I)$. The former relation gives $\mathcal{I}_{n}(m) \subseteq I$, and then the latter yields $\lambda_{n}(A)=\lambda_{n}(I) \geq \lambda_{n}\left(\mathcal{I}_{n}(m)\right)=\lambda_{n}(m)$. This proves the first assertion of the theorem.

For the second assertion, assume that $\lambda_{n}(A)=\lambda_{n}(m)=n K+i$. We also assume without loss of generality that $i \leq n-1$ (if $i=n$, then we can replace $K$ with $K+1$ ). Analyzing the argument above, we conclude that if the smallest among the projections $\left|\rho_{j}(A)\right|$ differed from the largest by at least 2 , then the size of the initial segment $I$ would satisfy the strict inequality $m<|I|$, implying $\lambda_{n}(A)=\lambda_{n}(I)>\lambda_{n}(m)$ in view of (27), since $\mathcal{I}_{n}(m)$ is closed, a contradiction. It follows that the largest of the projections $\left|\rho_{j}(A)\right|$ differs from the smallest one by at most 1 . Let $L$ denote the smallest of these projections, and let $k \in[0, n-1]$ be the number of indices $j \in[1, n]$ with $\left|\rho_{j}(A)\right|=L+1$ (so that there are $n-k$ those indices $j \in[1, n]$ with $\left.\left|\rho_{j}(A)\right|=L\right)$. From $n K+i=\lambda_{n}(A)=$ $(L+1) k+L(n-k)=n L+k$ and $k, i \in[0, n-1]$ we then conclude that $L=K$ and 
$k=i$. Thus, $A$ is contained in the Cartesian product of $i$ sets of size $K+1$ and $n-i$ sets of size $K$, and it is in fact equal to this product since $|A|=(K+1)^{i} K^{n-i}$.

In conclusion, we remark that the one-dimensional analog of Theorem 3 is the estimate

$$
\lambda_{n}(s m) \leq \lambda_{n-1}(m)+s, \quad m, s \geq 0 .
$$

The interested reader will easily verify that this estimate follows from the first assertion of Theorem 4 and, in fact, is equivalent to it.

\section{ACKNOWLEDGEMENT}

We thank Ben Barber and Larry Harper who have kindly helped us to rectify our ignorance about the edge-isoperimetric inequalities.

\section{REFERENCES}

[BL91] B. Bollobás and I. Leader, Edge-isoperimetric inequalities in the grid, Combinatorica 11 (4) (1991), 299-314.

[CGG16] S. Campi, R.J. Gardner, and P. Gronchi, Reverse and dual Loomis-Whitney-type inequalities, Trans. Amer. Math. Soc. 368 (7) (2016), 5093-5124.

[GMR10] K. Gyarmati, M. Matolcsi, and I.Z. Ruzsa, Superadditivity and submultiplicativity property for cardinalities of sumsets, Combinatorica 30 (2) (2010), 163-174.

[H04] L.H. HARPER, Global Methods for Combinatorial Isoperimetric Problems, Cambridge Studies in Advanced Mathematics, 90. Cambridge University Press, Cambridge, 2004.

[LW49] L.H. LoOmis and H. Whitney, An inequality related to the isoperimetric inequality, Bull. Amer. Math. Soc. 55 (1949), 961-962.

E-mail address: seva@math.haifa.ac.il

Department of Mathematics, The University of Haifa at Oranim, Tivon 36006, Israel

E-mail address: M.Rudnev@bristol.ac.uk

Department of Mathematics, University of Bristol, Bristol BS8 1TW, United KingDOM 